\title{
Comparison of the DeWitt metric in general relativity with the fourth-rank constitutive tensors in electrodynamics and in elasticity theory
}

\author{
Friedrich W. Hehl ${ }^{1,2, *}$ and Claus Kiefer ${ }^{1, \dagger}$ \\ ${ }^{1}$ Institute for Theoretical Physics, University of Cologne, \\ Zülpicher Str. 77, 50937 Köln, Germany \\ ${ }^{2}$ Department of Physics and Astronomy, University of Missouri, \\ Columbia, MO 65211, USA \\ file DWmetric_13.tex,07 Dec 2017
}

\begin{abstract}
We perform a short comparison between the local and linear constitutive tensor $\chi^{\lambda \nu \sigma \kappa}$ in four-dimensional electrodynamics (Sec.2), the elasticity tensor $c^{i j k l}$ in three-dimensional elasticity theory (Sec.3), and the DeWitt metric $G^{a b c d}$ in general relativity, with $a, b, \cdots=1,2,3$ (Sec.4). We find that the DeWitt metric has only six independent components.
\end{abstract}

Keywords: Canonical general relativity, DeWitt metric, premetric electrodynamics, elasticity theory, constitutive tensors

\section{Introduction}

The discovery of analogies between quantities in different areas of physics is of great importance. It can help to disclose similar mathematical structures and to

*E-mail: hehl@thp.uni-koeln.de

${ }^{\dagger}$ E-mail: kiefer@thp.uni-koeln.de 
build a conceptual bridge between physical theories. In this paper, we present such analogies which, to our knowledge, have largely remained unnoticed. The quantities in question are the DeWitt metric, which occurs in the canonical formalism of general relativity, and the constitutive tensors in electrodynamics and in elasticity theory.

Our paper is organized as follows. In Sec. 2, we review the basic properties for the constitutive tensor in electrodynamics and in Sec. 3 we review the properties of the elasticity tensor. The main part of our paper is Sec. 4. There we discuss analogies of the DeWitt metric with the tensors discussed in the earlier sections and point out the important property of the DeWitt metric that it has only six independent components.

\section{Four-dimensional electrodynamics}

The premetric Maxwell equations read [1,2] $d G=J$ and $d F=0$, where $G$ is the excitation two-form (consisting of the fields $\mathbf{D}$ and $\mathbf{H})^{1}$ and $F$ is the field strength two-form (consisting of the fields $\mathbf{E}$ and $\mathbf{B}$ ). In components, these equations read (see [3], with $\mu, \nu, \cdots=0,1,2,3$ ),

$$
\partial_{\nu} \mathfrak{G}^{\mu \nu}=\mathfrak{J}^{\mu}, \quad \partial_{\mu} F_{\nu \lambda}+\partial_{\nu} F_{\lambda \mu}+\partial_{\lambda} F_{\mu \nu}=0 .
$$

They result from electric charge conservation and magnetic flux conservation, respectively, and they are independent of any metric. Thus, only partial derivatives are required in (1). Since the fields $\mathfrak{G}^{\mu \nu}$ and $F_{\mu \nu}$ are unrelated so far, the system in (1) is not yet able to represent a predictive physical system.

In order to interrelate $\mathfrak{G}^{\mu \nu}$ and $F_{\mu \nu}$, we take the most general local and linear constitutive law

$$
\mathfrak{G}^{\lambda \nu}=\frac{1}{2} \chi^{\lambda \nu \sigma \kappa} F_{\sigma \kappa}
$$

The metric will play a role in this context of the constitutive law later, see below in (5). In (2), $\chi^{\lambda \nu \sigma \kappa}$ is a constitutive tensor density of rank 4 and weight +1 , with the dimension $[\chi]=[G] /[F]=1 /$ resistance [1]. ${ }^{2}$ Since both $\mathfrak{G}^{\lambda \nu}$ and $F_{\sigma \kappa}$ are antisymmetric in their indices, we have $\chi^{\lambda \nu \sigma \kappa}=-\chi^{\lambda \nu \kappa \sigma}=-\chi^{\nu \lambda \sigma \kappa}$. An antisymmetric pair of indices corresponds, in four dimensions, to six independent components. Thus, the constitutive tensor can be considered as a $6 \times 6$ matrix with 36 independent components.

\footnotetext{
${ }^{1}$ In Ref.[1], the excitation is denoted by $G=G(\mathcal{D}, \mathcal{H})$.

${ }^{2}$ See, for example, [4] for a recent discussion of this tensor.
} 
A $6 \times 6$ matrix can be decomposed in its tracefree symmetric part (20 independent components), its antisymmetric part (15 components), and its trace (1 component). At the level of $\chi^{\lambda \nu \sigma \kappa}$, this decomposition under the general linear group $G L(4, R)$ is reflected in

$$
\begin{aligned}
& \chi^{\lambda \nu \sigma \kappa}={ }^{(1)} \chi^{\lambda \nu \sigma \kappa}+{ }^{(2)} \chi^{\lambda \nu \sigma \kappa}+{ }^{(3)} \chi^{\lambda \nu \sigma \kappa} . \\
& 36=20 \quad \oplus \quad 15 \oplus \begin{array}{lll}
1 & \oplus
\end{array}
\end{aligned}
$$

The third part, the axion part [2], is totally antisymmetric and as such proportional to the Levi-Civita symbol, ${ }^{(3)} \chi^{\lambda \nu \sigma \kappa}:=\chi^{[\lambda \nu \sigma \kappa]}=\widetilde{\alpha} \widetilde{\epsilon}^{\lambda \nu \sigma \kappa}$; note that $\widetilde{\alpha}$ is a pseudoscalar since $\widetilde{\epsilon}^{\lambda \nu \sigma \kappa}$ has weight +1 . Therefore, the weight of $\widetilde{\epsilon}^{\lambda \nu \sigma \kappa}$ is essential information. The second part, the skewon part, is defined according to ${ }^{(2)} \chi^{\mu \nu \lambda \rho}:=\frac{1}{2}\left(\chi^{\mu \nu \lambda \rho}-\chi^{\lambda \rho \mu \nu}\right)$.

If the constitutive equation can be derived from a Lagrangian, which is the case as long as only reversible processes are considered, then ${ }^{(2)} \chi^{\lambda \nu \sigma \kappa}=0$. The principal part ${ }^{(1)} \chi^{\lambda \nu \sigma \kappa}$ fulfills the symmetries ${ }^{(1)} \chi^{\lambda \nu \sigma \kappa}={ }^{(1)} \chi^{\sigma \kappa \lambda \nu}$ and ${ }^{(1)} \chi^{[\lambda \nu \sigma \kappa]}=$ $0 .{ }^{3}$ Then, in this case of reversibility,

$$
\begin{aligned}
\chi^{\lambda \nu \sigma \kappa} & ={ }^{(1)} \chi^{\lambda \nu \sigma \kappa}+{ }^{(3)} \chi^{\lambda \nu \sigma \kappa} . \\
21 & =20 \oplus 1 .
\end{aligned}
$$

Note that up to here, we have argued at the level of premetric electrodynamics, that is, no metric was involved nor the Lorentz group $S O(1,3)$. In the special case of a Riemannian spacetime in vacuum, we have the decomposition $\left(g:=\operatorname{det} g_{\rho \sigma}\right)$

$$
\chi^{\lambda \nu \sigma \kappa}=\sqrt{\frac{\varepsilon_{0}}{\mu_{0}}} \sqrt{-g}\left(g^{\lambda \sigma} g^{\nu \kappa}-g^{\nu \sigma} g^{\lambda \kappa}\right)+\widetilde{\alpha} \widetilde{\epsilon}^{\lambda \nu \sigma \kappa},
$$

with the electric and the magnetic constants $\varepsilon_{0}$ and $\mu_{0}$, respectively.

\section{Three-dimensional linear elasticity}

In linear elasticity theory for homogeneous bodies, the stress tensor $\sigma^{i j}=\sigma^{j i}$, with $i, j, \cdots=1,2,3$, is related to the strain tensor $\varepsilon_{k l}:=\partial_{(k} u_{l)}=\varepsilon_{l k}$ by Hooke's law,

$$
\sigma^{i j}=c^{i j k l} \varepsilon_{k l}
$$

\footnotetext{
${ }^{3}$ For symmetrization and antisymmetrization we use the notation of tensor calculus: $(\lambda \nu):=$ $\frac{1}{2 !}\{\lambda \nu+\nu \lambda\},[\lambda \nu]:=\frac{1}{2 !}\{\lambda \nu-\nu \lambda\}$, and corresponding generalizations for $\mathrm{p}$ indices, see [5].
} 
see, for instance, [6] or [7]. Here, $u_{l}$ is the displacement field and $c^{i j k l}$ the constant fourth-rank elasticity tensor.

Since stress and strain are symmetric tensors, the elasticity tensor obeys the symmetries

$$
c^{i j k l}=c^{j i k l}=c^{i j l k} .
$$

A symmetric second rank tensor has six independent components. Therefore, by collecting the indices $i$ and $j$ into an index pair and $k$ and $l$ likewise, the elasticity tensor can be thought of as a $6 \times 6$ matrix with 36 independent components.

Usually one assumes that the stress tensor can be derived from an elastic potential $W$ (also called "strain energy function"), that is,

$$
\sigma^{i j}=\frac{\partial W}{\partial \varepsilon_{i j}}, \quad \text { or } \quad c^{i j k l}=\frac{\partial^{2} W}{\partial \varepsilon_{i j} \partial \varepsilon_{k l}} .
$$

Then the first and the last pair of indices of $c^{i j k l}$ commute,

$$
c^{i j k l}=c^{k l i j},
$$

and the $6 \times 6$ matrix is symmetric and carries only $36-15=21$ independent components. Thus, as is well known, the elasticity tensor has 21 independent components.

For the first irreducible piece under the general linear group $G L(3, R)$, we have

$$
{ }^{(1)} c^{i j k l}:=c^{(i j k l)} ;
$$

it has $\left(\begin{array}{c}3+4-1 \\ 4\end{array}\right)=15$ independent components. Furthermore,

$$
{ }^{(2)} c^{i j k l}:=c^{i j k l}-{ }^{(1)} c^{i j k l} .
$$

Thus, finally, for the case with an elastic potential, we have the decomposition

$$
\begin{aligned}
c^{i j k l} & ={ }^{(1)} c^{i j k l}+{ }^{(2)} c^{i j k l} . \\
21 & =15 \oplus 6 .
\end{aligned}
$$

An analysis by means of the Young tableaux techniques guarantees that this decomposition is irreducible under the $G L(3, R)$, indeed. One should compare this with the electromagnetic case in (4).

In this first step of the decomposition of $c^{i j k l}$, we take the constitutive law (6) at its face value, linking two symmetric second-rank tensors by a fourth-rank tensor. This tensor can only be decomposed with respect to $G L(3, R)$. That already 
such a first step is physically helpful is shown by the fact that the vanishing of ${ }^{(2)} c^{i j k l}$ represents the Cauchy relations, which are fulfilled for certain crystals, see $[11,12]$, that is, ${ }^{(2)} c^{i j k l}$ plays a role in nature. If, additionally, we use the threedimensional metric of the underlying space, we can take traces, and a finer decomposition is possible under the three-dimensional rotation group $S O(3)$. This finer decomposition, which is, however, not interesting in our context-since we compare, after all, with the linear decomposition in (4) - has been investigated in detail in [8].

Since ${ }^{(2)} c^{i j k l}$ depends on six independent components, one can express it in terms of a symmetric second-rank tensor,

$$
\Delta_{m n}:=\frac{1}{4} \epsilon_{m i l} \epsilon_{n j k}^{(2)} c^{i j k l}=\Delta_{n m},
$$

see [9, Eq.(25)].

For isotropic elastic bodies, with the Lamé moduli $\lambda$ and $\mu$, see [6], we have

$$
c^{i j k l}=\lambda g^{i j} g^{k l}+\mu\left(g^{i k} g^{l j}+g^{i l} g^{j k}\right) .
$$

Then the decomposition (12) yields [9],

$$
\begin{aligned}
{ }^{(1)} c^{i j k l} & =(\lambda+2 \mu) g^{(i j} g^{k l)}, \\
{ }^{(2)} c^{i j k l} & =\frac{\lambda-\mu}{3}\left(2 g^{i j} g^{k l}-g^{i k} g^{l j}-g^{i l} g^{j k}\right),
\end{aligned}
$$

with $g \Delta_{i j}=\frac{\lambda-\mu}{2} g_{i j}$ and $g:=\operatorname{det} g_{k l}$. The piece ${ }^{(2)} c^{i j k l}$ can be called the nonCauchy part of the elasticity tensor, see [10], since the conditions ${ }^{(2)} c^{i j k l}=0$ are called the Cauchy relations of elasticity theory. The Cauchy relations are only fulfilled if (i) the interaction forces between the atoms or molecules of a crystal are central forces, as, for example, in rock salt, (ii) each atom or molecule is a center of symmetry, and (iii) the interaction forces between the building blocks of a crystal can be well approximated by a harmonic potential. In most elastic bodies this is not fulfilled at all, see $[11,12]$.

Pure Cauchy materials $\left({ }^{(1)} c^{i j k l} \neq 0,{ }^{(2)} c^{i j k l}=0\right)$ and pure non-Cauchy materials $\left({ }^{(1)} c^{i j k l}=0,{ }^{(2)} c^{i j k l} \neq 0\right)$ do not seem to exist in nature. There are even some plausibility arguments against pure non-Cauchy materials.

\section{DeWitt metric}

The fundamental dynamical variable in canonical general relativity is the threedimensional metric denoted here by $h_{a b}$. As discussed in detail by DeWitt [13], 
one can define a metric on the (unconstrained) configuration space of all such three-metrics; in his honor, this metric is called DeWitt metric. ${ }^{4}$ A detailed review can be found in [16].

The DeWitt metric is defined in terms of the inverse three-metric $h^{a b}$ as follows; see, for example, Eq.(4.25) in [16],

$$
G^{a b c d}=\frac{\sqrt{h}}{2}\left(h^{a c} h^{b d}+h^{a d} h^{b c}-2 h^{a b} h^{c d}\right),
$$

for $h:=\operatorname{det} h_{e f}$ and $a, b, \cdots=1,2,3$. Incidentally, $G^{a b c d}$ is a tensor density of weight +1 . We note that it represents the covariant form of the DeWitt metric, in spite of the indices to be in the upper position. In the ADM form of the action, the combination $G^{a b c d} K_{a b} K_{c d}$ appears, where $K_{a b}$ are the components of the extrinsic curvature.

According to (17, the DeWitt metric obeys $G^{a b c d}=G^{b a c d}=G^{a b d c}$ and, moreover, the two index pairs $a b$ and $c d$ are commutative: $G^{a b c d}=G^{c d a b}$. Therefore, $G^{a b c d}$ as well as the constitutive tensor $\chi^{\mu \nu \kappa \lambda}$ and the elasticity tensor $c^{i j k l}$ are symmetric bilinear forms on a six-dimensional real vector space. In turn, the irreducible decomposition of $G^{a b c d}$ under the $G L(3, R)$ should be analogous to (12):

$$
G^{a b c d}={ }^{(1)} G^{a b c d}+{ }^{(2)} G^{a b c d},
$$

with ${ }^{(1)} G^{a b c d}=G^{(a b c d)}$ (15 independent components). If we take the totally symmetric part of (17), we recognize that it vanishes:

$$
{ }^{(1)} G^{a b c d}=0 .
$$

Thus,

$$
G^{a b c d}={ }^{(2)} G^{a b c d}=\frac{\sqrt{h}}{2}\left(h^{a c} h^{b d}+h^{a d} h^{b c}-2 h^{a b} h^{c d}\right)
$$

carries only six independent components.

Clearly, Eq. (20) is similar to (16), up to a constant factor and the square root of the metric. In analogy to elasticity, we can map the DeWitt metric to a symmetric second-rank tensor according to

$$
\stackrel{G}{\Delta}_{a b}:=\frac{1}{4} \epsilon_{a k l} \epsilon_{b m n} \frac{1}{\sqrt{h}} G^{k m n l}=\frac{1}{8} \epsilon_{a k l} \epsilon_{b m n}\left(h^{k n} h^{m l}+h^{k l} h^{m n}-2 h^{k m} h^{n l}\right),
$$

\footnotetext{
${ }^{4}$ The fundamental configuration space of general relativity is superspace, the space of all threegeometries. The question of a metric on superspace is discussed, for example, in [14, 15].
} 
see $[9,11,12]$. The factor $1 / \sqrt{h}$ was introduced in order to make $G^{a b c d} / \sqrt{h}$ a tensor density of weight 0 , exactly analogous to ${ }^{(2)} c^{i j k l}$ in (13). The second term within the parentheses of (21) drops out because of its symmetry in $k l$. If we expand the remaining two terms, we find

$$
\begin{aligned}
\stackrel{G}{\Delta}_{a b} & =\frac{1}{8}\left(\epsilon_{a k l} \epsilon_{b m n} h^{k n} h^{m l}-2 \epsilon_{a k l} \epsilon_{b m n} h^{k m} h^{n l}\right)=\frac{1}{8 h}\left(E_{a k l} E_{b}{ }^{l k}-2 E_{a k l} E_{b}{ }^{k l}\right) \\
& =\frac{1}{4 h}\left(-h_{a b}-2 h_{a b}\right)=-\frac{3}{4 h} h_{a b},
\end{aligned}
$$

where the $\epsilon$ denote the Levi-Civita symbols (tensor densities) and the $E$ the corresponding tensors; see, for example, [17].

Accordingly, the densitized metric $h_{a b} / \sqrt{h}$ can be directly expressed in terms of the DeWitt metric,

$$
h_{a b} / \sqrt{h}=-\frac{1}{3} \epsilon_{a k l} \epsilon_{b m n} G^{k m n l},
$$

a truly amazing formula. It is straightforward to show that (20) and (23) correspond in elasticity to the isotropic case with, in some suitable units, the Lamé constants $\lambda=-1$ and $\mu=\frac{1}{2}$. Thus, the "compression modulus" $\mathcal{K}$ of the DeWitt metric is $\mathcal{K}:=\lambda+(2 / 3) \mu=-2 / 3$, a highly unconventional "material," which reacts to pressure with expansion.

The reciprocity relation of the DeWitt metric ([16], Eq.(4.26)) reads

$$
G^{a b c d} G_{c d e f}^{-1}=\delta_{(e}^{a} \delta_{f)}^{b} .
$$

The inverse DeWitt metric $G_{c d e f}^{-1}$ depends on the number of space dimensions, whereas (17) does not. Furthermore, we introduced the power to the -1 explicitly in order to remind ourselves that the reciprocal of the DeWitt metric $G_{a b c d}^{-1}$ cannot be computed by simply lowering the indices of the DeWitt metric $G^{a b c d}$, see [15].

Let us emphasize that the reciprocal of the DeWitt metric has all the (15+6) independent components. The meaning of this should be investigated. In contrast, in the case of electrodynamics with $\chi^{\lambda \nu \sigma \kappa}$ and in elasticity with $c^{i j k l}$, their reciprocals carry the analogous irreducible decompositions as their originals, that is, they provide no new information.

In canonical general relativity, the relation between the canonical geometrodynamical momentum $p^{a b}$ and the extrinsic curvature $K_{c d}$ reads $(G=$ gravitational constant, speed of light $c=1$ )

$$
p^{a b}=\frac{1}{16 \pi G} G^{a b c d} K_{c d}
$$


see [16], Eq.(4.63). This is a Hooke type law of superspace with the 'stress' $p^{a b}$ and the 'strain' $K_{c d}$. If such an interpretation made sense, superspace would constitute a pure non-Cauchy continuum, that is, a fairly exotic 'substance'. Incidentally, this is reminiscent of Sakharov's [18, p.171] "metrical elasticity" of space, here, however, applied to superspace. It would be like in a crystal, the elastic constants of which are determined by the underlying molecular interaction forces.

The DeWitt metric (17) can be generalized to a one-parameter family of metrics by $[14,19]$

$$
{ }_{\beta} G^{a b c d}=\frac{\sqrt{h}}{2}\left(h^{a c} h^{b d}+h^{a d} h^{b c}-2 \beta h^{a b} h^{c d}\right),
$$

where $\beta$ is any real number (thus, for general relativity, $\beta=1$ ). If we calculate the total symmetrization of this object, we find that it is proportional to $1-\beta$, which means that the case of general relativity is distinguished by having only six independent components for the DeWitt metric.

Demanding symmetry under permutations of $a b$ and of $c d$, the inverse of (26) is unique and can be written in the form

$$
{ }^{\alpha} G_{a b c d}^{-1}=\frac{1}{2 \sqrt{h}}\left(h_{a c} h_{b d}+h_{a d} h_{b c}-2 \alpha h_{a b} h_{c d}\right)
$$

where ${ }^{5}$

$$
\alpha+\beta=3 \alpha \beta
$$

(thus, for general relativity, we have $\alpha=1 / 2$ ). Its total symmetrization is proportional to $1-\alpha$ and consequently is non-vanishing for the general relativistic case. (It vanishes for the special case of two space dimensions.)

To summarize, we have disclosed in our paper some interesting mathematical analogies (and differences) between quantities from different areas of physics. Whether they point to a deeper physical connection (e.g. in the context of emergent gravity) remains to be seen.

\section{Acknowledgments}

We thank Masud Chaichian (Helsinki) for a reference to Sakharov's work in the context of the metrical elasticity of space. Moreover, we thank Yakov Itin

\footnotetext{
${ }^{5}$ In $d$ space dimensions, we have $\alpha+\beta=d \alpha \beta$.
} 
(Jerusalem), Yuri Obukhov (Moscow), and Domenico Giulini (Hannover) for detailed remarks and corrections to our algebra, and Nick Kwidzinski and Branislav Nikolić (both of Cologne) for helpful discussions.

\section{References}

[1] F. W. Hehl and Yu. N. Obukhov, Foundations of Classical Electrodynamics: Charge, Flux, and Metric (Birkhäuser, Boston, MA, 2003). 2

[2] F. W. Hehl, Y. N. Obukhov, J. P. Rivera and H. Schmid, Relativistic nature of a magnetoelectric modulus of $\mathrm{Cr}_{2} \mathrm{O}_{3}$ crystals: A four-dimensional pseudoscalar and its measurement, Phys. Rev. A 77, 022106 (2008); see also arXiv:0707.4407 [cond-mat.other]. 2, 3

[3] E. J. Post, Formal Structure of Electromagnetics - General Covariance and Electromagnetics (North Holland, Amsterdam, 1962, and Dover, Mineola, NY, 1997). 2

[4] S. Schuster and M. Visser, Effective metrics and a fully covariant description of constitutive tensors in electrodynamics; see also arXiv:1706.06280 [grqc]. 2

[5] J. A. Schouten, Tensor Analysis for Physicists, 2nd ed. reprinted (Dover, Mineola, NY, 1989). 3

[6] J. E. Marsden and T. J. R. Hughes, Mathematical Foundations of Elasticity (Prentice-Hall, Englewoods Cliffs, NJ, 1983). 4, 5

[7] A. Sommerfeld, Mechanik deformierbarer Medien, Vorlesungen über Theoretische Physik, Vol. II, 5th ed. (Akademische Verlagsgesellschaft Geest \& Portig, Leipzig, 1964). 4

[8] Y. Itin and F. W. Hehl, Irreducible decompositions of the elasticity tensor under the linear and orthogonal groups and their physical consequences, J. Phys.: Conf. Ser. 597, 012046 (2015); see also arXiv:1411.5104 [condmat.other]. 5

[9] F. W. Hehl and Y. Itin, The Cauchy relations in linear elasticity theory, J. Elasticity 66, 185-192 (2002); see also arXiv:cond-mat/0206175. 5, 7 
[10] Y. Itin and F. W. Hehl, The constitutive tensor of linear elasticity: its decompositions, Cauchy relations, null Lagrangians, and wave propagation, $J$. Math. Phys. 54, 042903 (2013); see also arXiv:1208.1041 [cond-mat.other]. 5

[11] S. Haussühl, The deviations of the Cauchy relations (in German: Die Abweichungen von den Cauchy-Relationen), Phys. kondens. Materie 6, 181-192 (1967). 5, 7

[12] S. Haussühl, Physical Properties of Crystals: An Introduction (Wiley-VCH, Weinheim, Germany, 2007). 5, 7

[13] B. S. DeWitt, Quantum theory of gravity. I. The canonical theory, Phys. Rev. 160, 1113-1148 (1967). 5

[14] D. Giulini, What is the geometry of superspace? Phys. Rev. D 51, 5630-5635 (1995); see also arXiv:gr-qc/9311017. 6, 8

[15] D. Giulini, Dynamical and Hamiltonian formulation of General Relativity, in Chapter 17 of A. Ashtekar and V. Petkov (Eds.): Springer Handbook of Spacetime (Springer Verlag, Dordrecht, 2014); see also arXiv:1505.01403 [gr-qc]. 6, 7

[16] C. Kiefer, Quantum Gravity, 3rd ed. (Oxford University Press, Oxford, UK, 2012). $6,7,8$

[17] I. S. Sokolnikoff, Tensor Analysis (Wiley, New York, 1951). 7

[18] A. D. Sakharov, Collected Scientific Works, D. ter Haar et al. (eds.), translated from the Russian (Dekker, New York, 1982). 8

[19] D. Giulini and C. Kiefer, Wheeler-DeWitt metric and the attractivity of gravity, Phys. Lett. A 193, 21-24 (1994); see also arXiv:gr-qc/9405040. 8 